\title{
An Information Theoretical Analysis of Nanoscale Molecular Gap Junction Communication Channel Between Cardiomyocytes
}

\author{
Deniz Kilinc, Student Member, IEEE, and Ozgur B. Akan, Senior Member, IEEE
}

\begin{abstract}
Molecular communication which is a promising paradigm to communicate at nanoscale is inspired by nature. One of the molecular communication methods in nature is the gap junction (GJ) communication between cardiomyocytes. The GJ communication is achieved by diffusion of ions through GJ channels between cardiomyocytes. Propagation of the cardiac action potential, is used for the transmission of information. For the first time in the literature, the GJ communication channel is modeled and analyzed from the information theoretical perspective to obtain the communication channel characteristics. For the information theoretical analysis, a closed form expression is derived for the capacity of the GJ communication channel. The channel capacity, propagation delay and information transmission rate are analyzed numerically. Results of the numerical analyses show that a decrease in the channel capacity, an increase in the propagation delay and either an increase or a decrease in the transmission rate are correlated with increased incidence of cardiac diseases. The method that we use and results that are presented in this paper may help in the investigation, diagnosis and treatment of cardiac diseases as well as help in the design of nanodevices communicating via GJ channels.
\end{abstract}

Index Terms-Gap junction, cardiomyocyte, molecular communication, cardiac disease.

\section{INTRODUCTION}

Nanoscale communication between nanodevices (e.g., engineered organisms or artificial devices) is a novel and interdisciplinary concept including nanotechnology, biotechnology and communication technology [1]. The construction of nanonetworks by interconnecting nanodevices expands the capabilities of single nanodevices by means of cooperation between them [2], [3]. The realization of the communication between nanodevices can be achieved through electromagnetic, acoustic or molecular communication [4]. The molecular communication concept which is defined as the information transmission using molecules is inspired by nature [5]-[8]. Several nature-inspired and theoretically modeled molecular communication techniques exist in the literature including the models proposed based on pheromones [5], flagellated bacteria and catalytic nanomotors [2], pollen and spores [5], Förster Resonance Energy Transfer [19], as well as morphogenesis [20] to provide communication of nanodevices.

The authors are with the Next-generation and Wireless Communications Laboratory (NWCL), Department of Electrical and Electronics Engineering, Koc University, Istanbul, 34450, Turkey (e-mail: \{dkilinc, akan\}@ku.edu.tr)

This work was supported in part by the Turkish Scientific and Technical Research Council under grant \#109E257, by the Turkish National Academy of Sciences Distinguished Young Scientist Award Program (TUBA-GEBIP), and by IBM through IBM Faculty Award.
A promising approach for further understanding of the information transmission in a molecular environment is to investigate the molecular communication from the information theoretical perspective [9], [10]. The molecular communication is well investigated and modeled from the information theoretical perspective. For example, in [11], the information transfer capacity of the molecular communication, in which propagation of signaling molecules is based on Brownian motion and the number of molecules transmitted or received represents the coded information, is evaluated. In [12], diffusion-based molecular communication is investigated, where information is carried on the frequency of signal molecules. Furthermore, in [13], [14], a diffusion-based molecular communication is analyzed including several factors affecting the capacity of the channel and an error compensation method for the reliable communication is proposed. In [15], molecular communication based on calcium signaling is investigated by developing an information theoretical model.

In this study, for the first time in the literature, the nanoscale molecular communication between ventricular cardiomyocytes has been modeled and analyzed based on the information theory to reveal its channel characteristics. The communication between the cardiomyocytes is achieved by the transmission of ions through channels in specialized structures of the cell membrane called gap junctions (GJs) [16]. This type of communication is called GJ communication. The GJ communication is one of the potential approaches that have been proposed for the communication of nanodevices. For example, both molecular communication system inspired by cell to cell communications through GJs [17] and molecular communication based on intercellular calcium signaling [18] propose utilization of the GJ communication channels for the communication in the nanoscale. However, these studies do not propose a solution for the problem of modeling and analyzing the GJ communication channel from the information theoretical perspective. In this study, we model and analyze the GJ communication channel based on the information theory.

The potential models for the GJ communication of nanodevices can be engineered from commonly used techniques such as GJ transfection of cultured HeLa cells with connexin coding DNAs [21] or injection of Xenopus oocytes with connexin coding RNAs [22]. Therefore, the construction of the GJ communication channel is an existing technique used widely in physiology experiments. Unlike most of the existing models in the literature, this study presents information theoretical model of an already experimented, physically realizable and hence, 


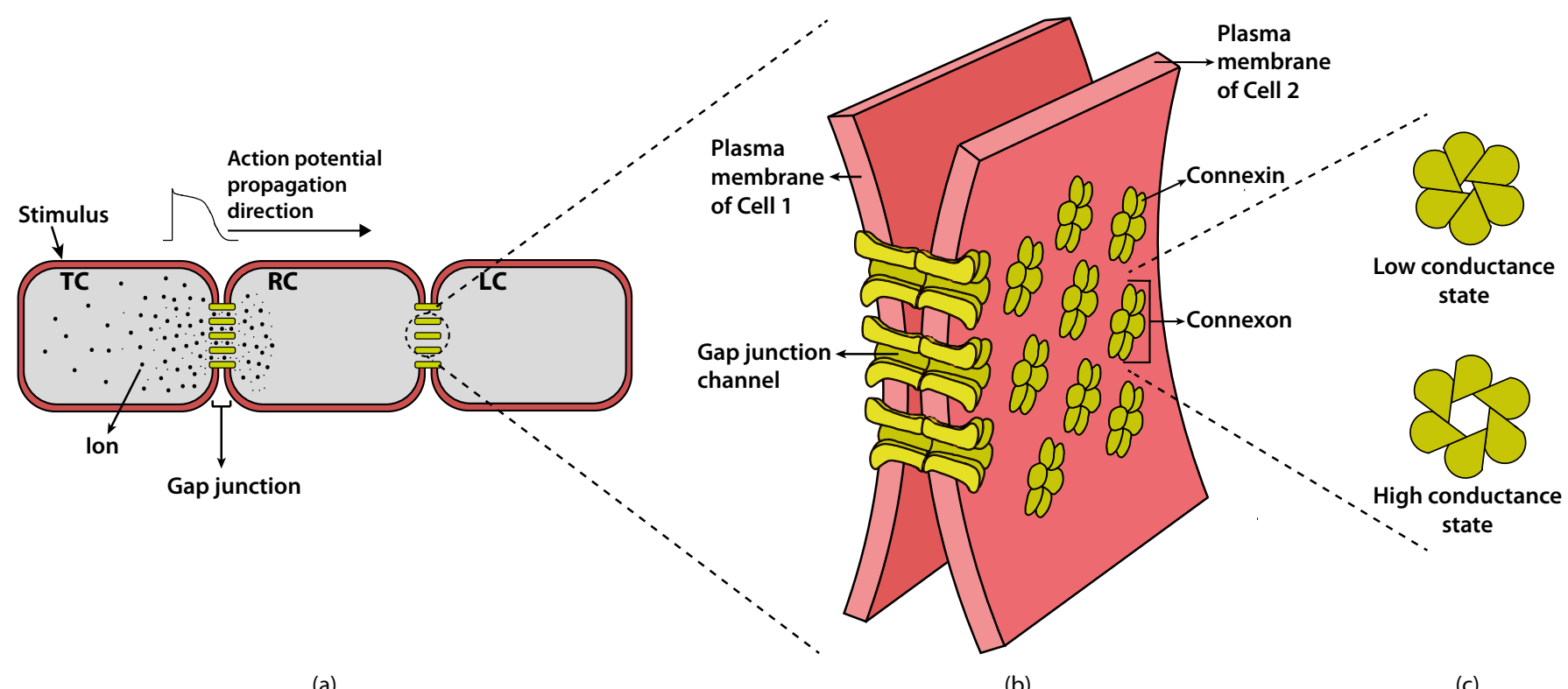

(a)

(b)

(c)

Fig. 1. A simplified model of the GJ communication and physical structure of GJs. (a) GJ communication model. A stimulus is applied to the transmitter cardiomyocyte (TC) in a cell chain and resulting AP starts to propagate towards the receiver cardiomyocyte (RC) by diffusion of ions through the GJ channels. The loading cardiomyocyte (LC) mimics loading effect of unstimulated myocytes connected to RC in cardiac fiber. (b) Enlarged section a GJ including several GJ channels. Membranes of two adjacent cells having hemichannels (connexons) interact to form complete GJ channels. (c) A slight twisting motion of connexon opens and closes the central pore of GJ channel; therefore, the GJ conductance changes accordingly.

realistic nanoscale communication channel. Since the channel is realizable, experimental studies can be conducted for model justification. The model presented in this paper may help in investigation, diagnosis and treatment of cardiac diseases. Furthermore, the information theoretical model can be used in physiology studies of the GJ communication channel to have a broad perspective in the investigations. Moreover, the model can be utilized in the design and engineering of the GJ communication for nanodevices.

The rest of the paper is organized as follows. Section II presents the physical model of the GJ communication. In Section III, an information theoretical model of the GJ communication channel is proposed. Section IV presents a numerical analysis of the channel capacity, the channel propagation delay and the information transmission rate with respect to several parameters. In Section V, relations between cardiac diseases and the channel capacity, the propagation delay and the information transmission rate are discussed. Finally, the concluding remarks are given in Section VI.

\section{Physical Model of Gap Junction COMMUNICATION}

The communication between two cardiomyocytes is achieved by the diffusion of ions at cell-to-cell contact domain called gap junction (GJ) through channels clustered in the GJ known as GJ channels [16]. In the myocardium, i.e., the muscular tissue of the heart, the nanoscale molecular communication between two cardiomyocytes is called GJ communication which is mediated by the GJ. For the rest of the paper, the GJ communication channel and the GJ channels should not be confused with each other since the former refers to the communication channel formed by a GJ while the latter refers to the physical channels clustered in a GJ. In Fig. 1(a), a GJ between two cardiomyocytes is indicated and in Fig. 1(b), GJ channels clustered in a GJ are shown.

In the GJ communication, the diffusion of ions between cardiomyocytes provides the propagation of the electrical activity which is called cardiac action potential (AP). The AP is initiated by the sinoatrial node, i.e., the impulsegenerating tissue of the heart, and is used for the transmission of information between cardiomyocytes [16]. That is, the information indicating whether cardiomyocytes contract or relax is encoded in the AP pulse train by the sinoatrial node. A successfully received AP pulse by a cardiomyocyte at rest causes this cardiomyocyte to contract, which is called as excitation-contraction coupling [23]. In contrast, when there is not any AP pulse received by a cardiomyocyte, corresponding cardiomyocyte stays at rest. Furthermore, cardiomyocytes have all-or-none property, i.e., if stimulus is above a threshold voltage, cardiomyocytes give complete response or otherwise they do not respond at all [24]. The contraction and relaxation of the heart as a single unit is the result of synchronous propagation of the APs.

A simplified model of the GJ communication is shown in Fig. 1(a). In the model, APs are transmitted by a single transmitter caridomyocyte (TC), propagated through the GJ by means of the diffusion of the ions, and received by a single receiver cardiomyocyte (RC). The ions diffused from TC to RC continue to diffuse to adjacent unstimulated cardiomyocytes which affects the AP initiation in RC. Then, to have a more realistic GJ communication model, there is also a loading 
cardiomyocyte (LC) to capture the loading effects of the unstimulated myocytes connected to RC in the cardiac fiber. TC, RC, and LC are identical. According to the properties of both cardiomyocytes and the AP propagation stated above, we model the GJ communication between cardiomyocytes as binary digital communication with on-off keyed (OOK) modulation. That is, an AP pulse is considered as binary bit 1 and its absence is considered as binary bit 0 .

\section{A. Action Potential Propagation}

The heart beat is generated in the form of electrical activity of the impulse-generating cells of the sinoatrial node. The initiation and the propagation of the electrical activity, i.e., the AP, is the most efficient molecular signal conduction mechanism in the myocardium [23]. The AP initiation in cardiomyocytes is described as following: if a stimulus causes the membrane potential, i.e., the potential difference across the membrane of the cardiomyocyte, to become less negative than a threshold level, the sodium $\left(\mathrm{Na}^{+}\right)$channels embedded in the membrane open and allow $\mathrm{Na}^{+}$ions to enter the cardiomyocyte. This makes the membrane potential to rise at a rate of about $300 \mathrm{~V} / \mathrm{sec}$ causing depolarization of the membrane. Such an rapid increase in the membrane potential is called upstroke of the AP. As the membrane voltage rises, $\mathrm{Na}^{+}$channels close due to a process called inactivation. Next, calcium $\left(\mathrm{Ca}^{2+}\right)$ and potassium $\left(\mathrm{K}^{+}\right)$channels open which causes the membrane potential stay constant; that is, the inward $\mathrm{Ca}^{2+}$ current and the outward $\mathrm{K}^{+}$current are the same for a while. Then, $\mathrm{Ca}^{2+}$ channels close but $\mathrm{K}^{+}$channels are still open which causes rapid repolarization of the membrane. Finally, the membrane potential is restored to around $-85 \mathrm{mV}$. A significant amount of current knowledge about the action potential generation process comes from the studies presented in [25]-[27]. For this study, we use the cardiac ventricular action potential model given in [26].

The potential difference between the stimulated and the unstimulated adjacent cardiomyocytes causes the ions to drift through the GJ channels leading the synchronous propagation of the AP. That is, the GJ coupling enables the wave of the action potential propagate from one cardiomyocyte to neighbor cardiomyocyte by means of diffusion of ions through the GJ channels. The AP propagation model that we use is presented in [28]. The propagation model mathematically equivalent to assume that each cardiomyocyte is isopotential, i.e., the membrane potential of each individual cardiomyocyte is the same over its membrane surface and that according to the model, the most important factor in determining the propagation is delay for an AP to get through GJs. For the ion current flowing through the cardiomyocyte membrane, the current balance equation is

$$
C_{m} S \frac{d v_{n}}{d t}=\frac{1}{R_{g j}}\left(v_{n+1}-2 v_{n}+v_{n-1}\right)+S I_{m}
$$

where $v_{n}$ is the membrane potential for the $n$th cell in one dimensional cardiac fiber, i.e., the potential difference between inside and outside of the cardiomyocyte, $C_{m}$ is the membrane capacitance per unit area of the cardiomyocyte membrane,
$S$ is the surface area of the membrane, $R_{g j}$ is the total GJ resistance between the cardiomyocytes, and $I_{m}$ specifies the ionic currents, e.g., $\mathrm{Na}^{+}, \mathrm{Ca}^{2+}$, and $\mathrm{K}^{+}$currents, per unit area of the membrane described in detail in [26], [27]. We use the piecewise linear approximation of $I_{m}$ given in [28] as

$$
I_{m}(v)= \begin{cases}\frac{\alpha_{1}\left(V_{1}-v\right)}{R_{m}}, & v<V^{*} \\ \frac{\alpha_{2}\left(V_{2}-v\right)}{R_{m}}, & V^{*}<v\end{cases}
$$

where $R_{m}$ is the membrane resistivity, $V_{1}$ is the resting potential of the cardiomyocyte membrane, $V_{2}$ is the sodium equilibrium potential, $V^{*}$ is the activation threshold voltage for $\mathrm{Na}^{+}$current, and $\alpha_{1}$ and $\alpha_{2}$ are constants chosen properly such that for $V_{1}<v<V_{2}$, area under both the piecewise linear function and the approximated function are the same [28]. The parameter values of (2) are taken as $V_{1}=-87 \mathrm{mV}$, $V_{2}=28.8 m V, V^{*}=-34.8 m V, \alpha_{1}=1$, and $\alpha_{2}=108$ from the results of the AP model based on the formalism presented in [29], [30]. The piecewise linear function with the given numerical values captures the numerical features of the dynamics of the AP upstroke [28].

The most important deduction that can be extracted from (1) is that the propagation fails if $R_{g j}$ is sufficiently large. That is, an increase in $R_{g j}$ causes a decrease in the number of the diffused ions which may not be enough to increase the membrane potential of $\mathrm{RC}$ to the threshold value. The critical GJ resistance between TC and RC is denoted by $R_{g j}^{*}$ and $\mathrm{RC}$ has infinite voltage threshold value to be excited for $R_{g j}>R_{g j}^{*}$. In [28], an expression is given for $R_{g j}^{*}$ as

$$
\begin{aligned}
R_{g j}^{*}=L \sqrt{\frac{\Gamma R_{m}}{S}} & {\left[\frac{E_{2}^{2}-2 \mu_{2} E_{2}+1}{\left(1-E_{2}^{2}\right) \sqrt{\alpha_{2}}}\right.} \\
& \left.+\left(\frac{V_{2}-V^{*}}{V_{1}-V^{*}}\right) \frac{E_{1}^{2}-2 \mu_{1} E_{1}+1}{\left(1-E_{1}^{2}\right) \sqrt{\alpha_{1}}}\right]
\end{aligned}
$$

where $L$ is the length of the cardiomyocyte, and $\Gamma, E_{1}$, and $E_{2}$ are variables defined as

$$
\begin{aligned}
\Gamma & =\left(R_{i} / V_{i}\right)+\left(R_{e} / V_{e}\right) \\
E_{j} & =\exp \left(\sqrt{\left(\alpha_{j} \Gamma L^{2} S\right) / R_{m}}\right), \quad j=1,2
\end{aligned}
$$

where $R_{i}$ and $R_{e}$ are the cytoplasmic and extracellular medium resistivity values, respectively, and, $V_{i}$ is the volume of the cytoplasm of the cardiomyocyte and $V_{e}$ is the volume of the extracellular medium that surrounds the cardiomyocyte in the myocardium. The values of $\alpha_{1}, \alpha_{2}, V_{1}, V_{2}$, and $V^{*}$ that we use in our work are as the same as the values given above and $\mu_{1}$ and $\mu_{2}$ satisfy the equality $R_{g j}^{*}=R_{g j}$ where $R_{g j}$ is

$$
R_{g j}=2 L \sqrt{\frac{\Gamma R_{m}}{\alpha_{j} S} \frac{\left(\mu_{j}-\frac{1}{E_{j}}\right)\left(\mu_{j}-E_{j}\right)}{\mu_{j}\left(E_{j}-\frac{1}{E_{j}}\right)}}, j=1,2 .
$$

Numerical result of $R_{g j}^{*}$ is found by solving two nonlinear equations which are $R_{g j}^{*}=R_{g j}$ for $j=1$ and $R_{g j}^{*}=R_{g j}$ for $j=2$. The upper limit of the GJ resistance for successful 
propagation is $R_{g j}^{*}$ since the AP propagation strictly fails if $R_{g j}>R_{g j}^{*}$ as stated above.

\section{B. Physical Model of Gap Junction Channels}

The GJs include GJ channels that form a connection between neighboring cells in many tissues and organs providing chemical and electrical molecular communication [16]. The length and diameter of a complete GJ channel is approximately $10-15 \mathrm{~nm}$ and $2-3 \mathrm{~nm}$, respectively [31]. Therefore, the GJ communication takes place at nanoscale. The physical structure of a GJ channel is formed by two hemichannels called connexons which are embedded in the membrane of the connected cells. Furthermore, each hemichannel includes six transmembrane proteins called connexins that are oligomerized in a hexameric structure to form a pore. Two hemichannels belonging to adjacent cells interact in order to form a complete GJ channel. The structure of GJ channels in a GJ can be seen in Fig. 1(b). GJ channels have an evident selectivity for particle passage based on the permeability of the GJ channels and size of the particles. Thus, the AP propagation between cardiomyocytes depends on the permeability of the GJ channels since the diffusion of the ions is affected by the permeability of the GJ channels [31]

According to the stochastic behavior of GJ channels in a GJ [32], a GJ channel have two voltage-sensitive gates in series, one located in each hemichannel which controls the GJ permeability. Each gate has two states, an open state resulting in a high conductance value and a closed state resulting in a low conductance value for a hemichannel [32] as indicated in Fig. 1(c). The voltage-sensitive gates control the channel conductance state in accordance of junctional voltage, i.e., potential difference between the membranes potentials of two adjacent cardiomyocytes denoted by $V_{j}$, in a stochastic manner. Hence, the gates associated with each connexon can be used in order to describe the stochastic GJ channel dynamics. At any instant a GJ channel can be in any one of four states:

- HH state: both gates are open

- HL state: one gate is open and the other gate is closed

- LH state: one gate is open and the other gate is closed

- LL state: both gates are closed

The occurrence of the LL state, however, is very rare [33] and such a state has a very small conductance compared with the other states; thus, we choose to include only the first three states by neglecting LL state. The stochastic dynamics of the GJ channels given in [32] is described by probabilities of a channel being in any of HH, HL and LH states, denoted by $p_{H H}, p_{H L}$, and $p_{L H}$, respectively, given as follows

$$
\begin{gathered}
p_{H H}+p_{L H}+p_{H L}=1 \\
\frac{d p_{L H}}{d t}=\beta_{1}\left(V_{j}\right) \cdot p_{H H}-\alpha_{1}\left(V_{j}\right) \cdot p_{L H} \\
\frac{d p_{H L}}{d t}=\beta_{2}\left(V_{j}\right) \cdot p_{H H}-\alpha_{2}\left(v_{j}\right) \cdot p_{H L}
\end{gathered}
$$

where $\alpha_{1}\left(V_{j}\right), \alpha_{2}\left(V_{j}\right), \beta_{1}\left(V_{j}\right)$ and $\beta_{2}\left(V_{j}\right)$ are rate constants of the state transitions as demonstrated in Fig. 2. The rate

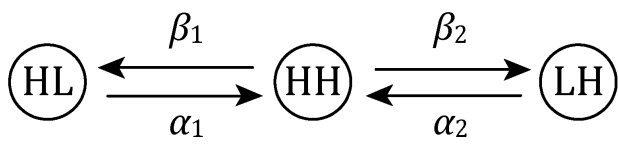

Fig. 2. The kinetic behavior of a GJ channel modeled by a state machine with three states, i.e., HH, HL, and LH. The rate constants $\beta$ and $\alpha$ refer to the forward reactions high to low and the backward reactions low to high, respectively.

constants are given as

$$
\begin{aligned}
& \alpha_{1}\left(V_{j}\right)=\lambda \exp \left(-A_{\alpha}\left(V_{j}-V_{0}\right)\right) \\
& \alpha_{2}\left(V_{j}\right)=\lambda \exp \left(A_{\alpha}\left(V_{j}+V_{0}\right)\right) \\
& \beta_{1}\left(V_{j}\right)=\lambda \exp \left(A_{\beta}\left(V_{j}-V_{0}\right)\right) \\
& \beta_{2}\left(V_{j}\right)=\lambda \exp \left(-A_{\beta}\left(V_{j}+V_{0}\right)\right)
\end{aligned}
$$

where $V_{0}$ is the junctional voltage at which the opening and closing rates of the GJ channel gates have the same common value $\lambda$, and $A_{\alpha}$ and $A_{\beta}$ are constants that indicate the sensitivity of a GJ channel to the junctional voltage differences [32]. Since connexin43 GJ protein is the major GJ protein in the ventricular muscle [34], in numerical analysis, we use data obtained experimentally for connexin 43 GJ protein. Thus, we use the constants as $\lambda=0.69, A_{\alpha}=0.04(m V)^{-1}, A_{\beta}=$ $0.07(m V)^{-1}$, and $V_{0}=62 m V$ adopted from experimental studies of connexin43 GJ protein [35].

\section{Action Potential Propagation Failure}

The conductance of each GJ channel, denoted by $G_{c h}$, is a random variable whose probability mass function is

$$
f_{G}\left(G_{c h}\right)= \begin{cases}p_{H H}, & \text { if } G_{c h}=G_{H H} \\ p_{H L}, & \text { if } G_{c h}=G_{H L} \\ p_{L H}, & \text { if } G_{c h}=G_{L H}\end{cases}
$$

where $G_{H H}, G_{H L}$, and $G_{L H}$ are the conductance values of a single GJ channel in the respective state [32]. In this study, we use the GJ channel conductance values as $G_{H H}=73 p S$, $G_{H L}=12 p S$, and $G_{L H}=12 p S$, which are experimental results for connexin43 type GJ protein [34]. Furthermore, since the GJ channels in a GJ are connected in parallel, the total conductance of the GJ which have $n_{H H}, n_{H L}$, and $n_{L H}$ GJ channels in $\mathrm{HH}, \mathrm{HL}$, and $\mathrm{LH}$ states, respectively, is given as

$$
G_{g j}\left(V_{j}\right)=n_{H H} \cdot G_{H H}+n_{H L} \cdot G_{H L}+n_{L H} \cdot G_{L H}
$$

For a GJ consisting of $N$ GJ channels, probability that there are $n_{H H}, n_{H L}$, and $n_{L H}$ GJ channels in $\mathrm{HH}, \mathrm{HL}$, and LH states, respectively, is found by multinomial distribution function which is given by

$$
\begin{aligned}
& \operatorname{Pr}\left\{G_{g j}\left(V_{j}\right)=n_{H H} \cdot G_{H H}+n_{H L} \cdot G_{H L}+n_{L H} \cdot G_{L H}\right\} \\
& = \begin{cases}N ! \frac{p_{H H}^{n_{H H}} \cdot p_{L H}^{n_{L H}} \cdot p_{H L}^{n_{H L}}}{n_{H H} ! \cdot n_{L H} ! \cdot n_{H L} !}, & \text { if } n_{H H}+n_{L H}+n_{H L}=N \\
0, & \text { otherwise }\end{cases}
\end{aligned}
$$




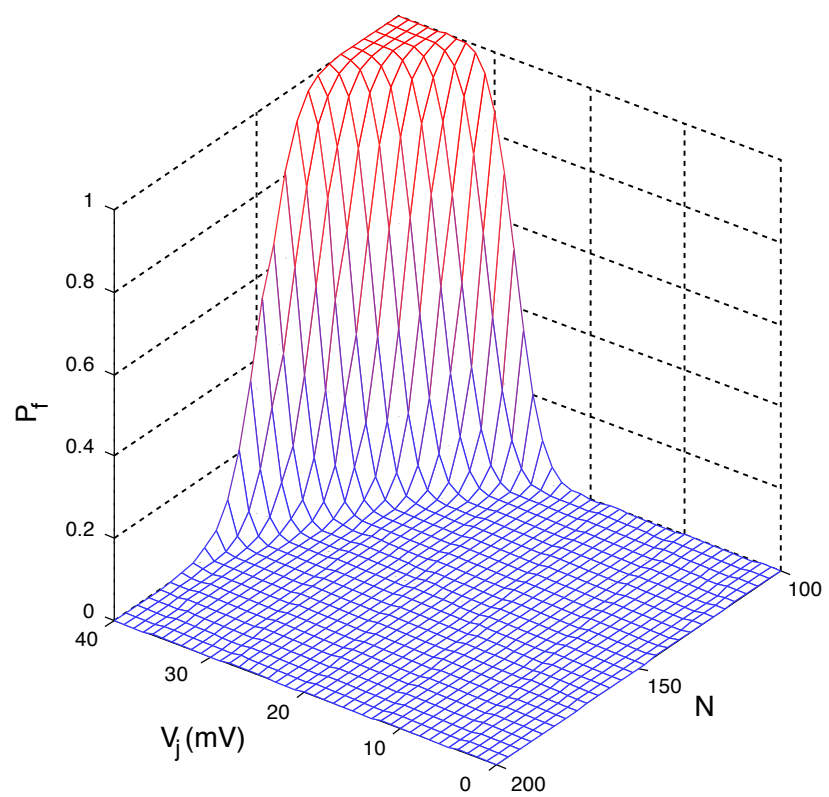

Fig. 3. $P_{f}$ plot with respect to junctional voltage, $V_{j}$, and the total number of the GJ channels in a GJ, $N$. For other parameters, the typical physiological values are used which are $L=100 \mu \mathrm{m}, r=11 \mu \mathrm{m}, R_{i}=250 \Omega \mathrm{cm}$, $R_{e}=100 \Omega \mathrm{cm}, R_{m}=20 \mathrm{k} \Omega \mathrm{cm}^{2}$, and $V_{e}=V_{i} / 5$ adopted from [26], [40].

Assume that a GJ includes $N$ GJ channels and they are distributed such that the number of the GJ channels in $\mathrm{HH}, \mathrm{HL}$, and LH states are $n_{H H}, n_{H L}$, and $n_{L H}$, respectively. Then, the total number of different combinations of $n_{H H}, n_{H L}$, and $n_{L H}$ satisfying $n_{H H}+n_{L H}+n_{H L}=N$ can be found in the following manner. Firstly, selecting $n_{H H}=N, n_{H L}=0$, and $n_{L H}=0$ gives one combination. Secondly, for $n_{H H}=N-1$, there are two possible combinations: $n_{H L}=0, n_{L H}=1$ and $n_{H L}=1, n_{L H}=0$. Next, for $n_{H H}=N-2$, there are three possible combinations: $n_{H L}=0, n_{L H}=2, n_{H L}=1$, $n_{L H}=1$, and $n_{H L}=2, n_{L H}=0$. If we continue in similar way, for $n_{H H}=0$, there are $(N+1)$ combinations. As a result, the total number of different combinations is given by $1+2+$ $3+\ldots+(N+1)=\frac{1}{2}(N+1)(N+2)$. Furthermore, for each combination, the total conductance value and the probability of the corresponding combination is given in (11) and (12), respectively. We use MATLAB to obtain the probability mass function of the total conductance of a GJ including total $N$ GJ channels by evaluating all possible channel state combinations and the corresponding probabilities.

Since the propagation of the AP fails when the GJ conductance value, $G_{g j}\left(V_{j}\right)$, is less than the critical conductance value, $G_{g j}^{*}$, we can find the probability of the propagation failure, denoted by $P_{f}$, using the probability mass function of the GJ conductance as follows

$$
P_{f}=\operatorname{Pr}\left\{G_{g j}\left(V_{j}\right)<G_{g j}^{*}\right\}
$$

where the critical conductance value is reciprocal of the critical resistance value, $G_{g j}^{*}=1 / R_{g j}^{*}$. The failure probability $P_{f}$ depends on several parameters such as the critical GJ conductance $G_{g j}^{*}$, the junctional voltage $V_{j}$, and the number of the GJ channels $N$, as clearly observed in (8), (12), and (13). In Fig. 3, variation of $P_{f}$ with respect to $N$ and $V_{j}$ is shown.
For constant $N$, increasing $V_{j}$ does not severely affect $P_{f}$ at low $V_{j}$; however, for $V_{j}>30 \mathrm{mV}$, increasing $V_{j}$ significantly increases $P_{f}$. The increase in $P_{f}$ at high $V_{j}$ is due to decreased GJ conductance which is caused by increased closure rates and decreased opening rates of the GJ hemichannels for $V_{j}>V_{0}$ as given in (9). Therefore, at higher $V_{j}, P_{f}$ is higher than $P_{f}$ at low $V_{j}$. Moreover, at constant $V_{j}$, increasing $N$ decreases $P_{f}$ as seen in Fig. 3 because conductance of the GJ increases with increasing $N$ as given in (11).

\section{Spontaneous Action Potential Initiation}

The AP initiation in a cardiomyocyte is caused by the diffusion of the ions from the stimulated cardiomyocte to the unstimulated neighbor cardiomyocyte through the GJ channels in the GJ. That is, the diffusion of ions stimulates the neighbor cardiomyocyte. However, the AP generation can be triggered in the absence of a stimulus due to some abnormalities of cardiomyocytes which are early afterdepolarizations (EADs) and delayed afterdepolarizations (DADs) [27]. Although normal and healthy ventricular cardiomyocytes do not exhibit such spontaneous generation of the AP [36], we incorporate the spontaneous AP generation into the GJ communication model considering diseased cardiomyocytes.

The spontaneous AP initiation in a cardiomyocyte can be triggered by the $\mathrm{Ca}^{2+}$ release units (CRUs) of the sarcoplasmic reticulum [37]. If a CRU fires, it emits a $\mathrm{Ca}^{2+}$ ion spark into the cytoplasm of the cardiomyocyte. The emitted $\mathrm{Ca}^{2+}$ ions increase the membrane potential of the cardiomyocyte. Therefore, if the membrane potential becomes greater than the threshold due to the released $\mathrm{Ca}^{2+}$ ions by CRUs, an AP is triggered in the cardiomyocyte without an external stimulus. The probability that a CRU fires in the time duration $T_{s}$ is

$$
P_{s}=1-\exp \left(-\frac{P_{\max } \rho^{n}}{K^{n}+\rho^{n}} T_{s}\right)
$$

where $P_{\max }$ is the maximum probability of $\mathrm{Ca}^{2+}$ spark occurrence/CRU/unit time, $\rho$ is the free $\mathrm{Ca}^{2+}$ molar concentration in the cytoplasm, $K$ is the $\mathrm{Ca}^{2+}$ sensitivity parameter and $n$ is the Hill coefficient [37]. Let the number of the CRUs that fire in time $T_{s}$ be $m$. Assuming the CRUs are independent of each other, $m$ is distributed by the binomial probability distribution as $m \sim \mathrm{B}\left(M, P_{s}\right)$ where $M$ is the total number of the CRUs in a cardiomyocyte. That is,

$$
\operatorname{Pr}\{m \text { sparks }\}=\left(\begin{array}{c}
M \\
m
\end{array}\right) P_{s}^{m}\left(1-P_{s}\right)^{M-m}
$$

Since $M$ is a large number, we can use the Gaussian approximation of the binomial distribution given in (15) as

$$
m \sim \mathcal{N}\left(M P_{s}, M P_{s}\left(1-P_{s}\right)\right)
$$

One $\mathrm{Ca}^{2+}$ spark does not release enough $\mathrm{Ca}^{2+}$ to initiate an AP. In the GJ communication model, we use the half of the number of the CRUs as the required number of sparks to trigger an $\mathrm{AP}$ in $T_{s}$ duration. Thus, the probability of an AP wave initiation without any stimulus, denoted by $P_{w}$, is

$$
P_{w}=\operatorname{Pr}\left\{\frac{M}{2}<m\right\}=Q\left(\frac{(M / 2)-M P_{s}}{M P_{s}\left(1-P_{s}\right)}\right)
$$




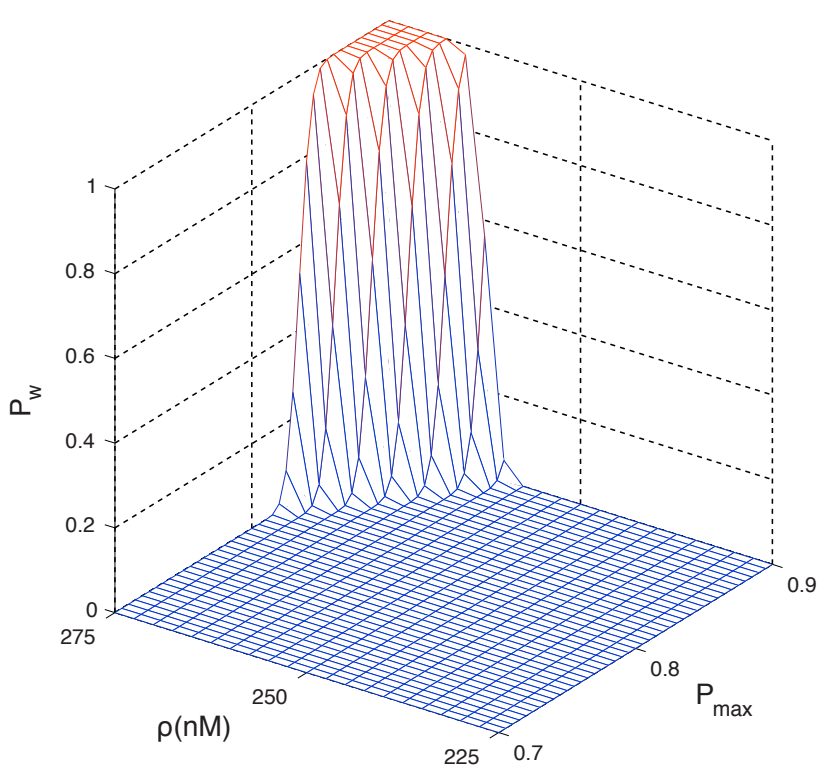

Fig. 4. $P_{w}$ plot with respect to $\rho$ and $P_{\max }$. For the other parameters, $K=15 \mu M, M=30000, n=1.6$, and $T_{s}=500 \mathrm{msec}$ [37]

where $Q(\cdot)$ is the $Q$-function. Since the distribution of $m$ is approximated by Gaussian distribution, we can use $Q$-function to describe the probability given in (17). In Fig. $4, P_{w}$ with respect to both $\rho$ and $P_{\max }$ is plotted. An increase in $P_{\max }$ causes an increase in $P_{s}$ given in (14). Thus, an increase in $P_{\max }$ causes an increase in the expected number of the emitted $\mathrm{Ca}^{2+}$ sparks which increases the probability of the initiation of an AP $P_{w}$ seen in Fig. 4. Furthermore, since increasing the free $\mathrm{Ca}^{2+}$ concentration $\rho$ in the cardiomyocyte cytoplasm increases $P_{s}$, an increase in $\rho$ due to concentration fluctuations increases the spontaneous AP initiation probability.

\section{INFORMATION THEORETICAL ANALYSIS OF GAP JUNCTION COMMUNICATION CHANNEL}

We investigate the GJ communication channel between TC and RC as illustrated in Fig. 1(a) for an information theoretical model of the GJ communication channel. The cardiac APs are used for information transmission between TC and RC. The AP pulse represents bit 1 and absence of the AP pulse represents bit 0 . For the information theoretical model, we consider two cases to figure out transmission probabilities of the information from TC to RC. The first case is the failure of the AP transmission; that is, $\mathrm{RC}$ detects bit 0 while bit 1 is transmitted by TC. The probability of the first case is $P_{f}$ given in (13). The second case is the initiation of the AP at RC in the absence of a stimulus caused by TC; that is, RC detects bit 1 while bit 0 is transmitted by TC. The probability of the second case is $P_{w}$ given in (17). The AP propagation failure and the spontaneous AP initiation are caused by the noise factors that intrinsically arise in the GJ communication. The intrinsic noise factors are related to the stochastic nature of both the GJ channels and the CRUs. Therefore, the information theoretical channel model of the GJ communication includes the peculiar noises and noise factors specific to the GJ communication.

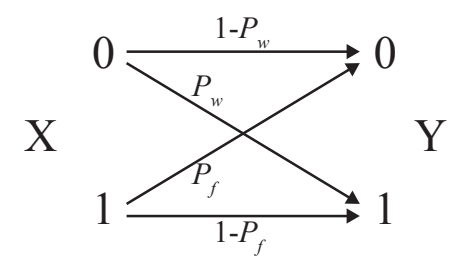

Fig. 5. The GJ communication channel transmission diagram with the transmission probabilities. The communication channel expresses binary asymmetric channel (BAC) characteristics.

The GJ communication channel exhibits binary asymmetric channel (BAC) characteristics with on-off keyed (OOK) modulation which is represented in Fig. 5 based on the analysis that we introduced so far. The communication channel also has memoryless characteristics because of the fact that when the membrane voltage returns to its steady state value at the end of an AP transmission, the voltage dependent state probabilities of the GJ channels returns also their steady state values. Therefore, afterwards if another AP is transmitted, initial condition for the probabilities of the GJ channel states are the same as the previous transmission.

Assume that $X$ is the transmitted bit by TC and $Y$ is the received bit by RC. Then, we define the AP transmission probability of TC as $P_{A P}$, i.e.,

$$
P(x)= \begin{cases}P_{A P}, & \text { if } X=1 \\ 1-P_{A P}, & \text { if } X=0\end{cases}
$$

Using (18) and the channel transmission probabilities seen in Fig. 5, the joint probability distribution of $X$ and $Y$ denoted by $P(x, y)$ is given as

$$
P(x, y)= \begin{cases}\left(1-P_{A P}\right)\left(1-P_{w}\right), & \text { if }(X=0, Y=0) \\ \left(1-P_{A P}\right) P_{w}, & \text { if }(X=0, Y=1) \\ P_{A P} P_{f}, & \text { if }(X=1, Y=0) \\ P_{A P}\left(1-P_{f}\right), & \text { if }(X=1, Y=1)\end{cases}
$$

The joint distribution $P(x, y)$ expresses the probability of that the observed output symbol is $y$ and the transmitted symbol is $x$. The mutual information between $X$ and $Y$ denoted by $I(X ; Y)$ is given in [38] as follows

$$
I(X ; Y)=\sum_{x, y} P(x, y) \log _{2} \frac{P(x, y)}{P(x) P(y)}
$$

where $P(y)$ is the probability distribution of $Y$. Obtaining $P(y)$ is straightforward using the joint distribution $P(x, y)$. The GJ communication channel capacity denoted by $C_{g j}$ is the maximum value of the mutual information [38] and the capacity of the GJ communication channel is found as follows

$$
\begin{aligned}
C_{g j}= & \max _{P(x)} I(X ; Y) \\
= & \frac{\left(1-P_{w}\right) H\left(P_{f}\right)-P_{f} H\left(1-P_{w}\right)}{P_{f}+P_{w}-1} \\
& \quad+\log _{2}\left(1+2 \frac{H\left(1-P_{w}\right)-H\left(P_{f}\right)}{P_{f}+P_{w}-1}\right)
\end{aligned}
$$


where $H(\cdot)$ is the binary entropy function defined as

$$
H(p)=-p \log _{2} p-(1-p) \log _{2}(1-p)
$$

The channel characteristics of the GJ communication varies with respect to several parameters such as the number of the GJ channels in the GJ, the membrane resistivity, the cell length and radius. Since such physiological properties vary between individual organisms and even between different sites on the same heart, in the next section, we analyze the GJ communication channel characteristics with respect to several physiological parameters.

\section{NUMERICAL ANALYSIS}

In this section, we present a numerical analysis of the GJ communication parameters that are the channel capacity, the channel propagation delay and the information transmission rate to show how these parameters change with respect to the properties of cardiomyocytes and GJs. The results of the analysis can be used in investigation of biological properties of GJ coupled cardiomyocytes as well as in the design of nanodevices that use the GJ communication channel.

For the numerical analysis, we model the cardiomyocyte membranes using the model presented in [26]. The propagation of the AP is simulated in a 3-cell fiber as illustrated in Fig. 1(a). A stimulus is applied to TC in order to start the AP propagation. After exciting TC, an AP propagates to RC through the GJ between. The coupling GJ channels behave as described in Section II. Since the occurrence probabilities of the GJ channel states depend on the junctional voltage between cardiomyocytes, we simulate the AP propagation by using the expected values of the total GJ conductance. For discrete time steps $\Delta t=0.01 \mathrm{~ms}$, the junctional voltage is found from difference between the membrane voltages of TC and RC. Then, at the obtained junctional voltage, the expected value of the total conductance is evaluated and changed with the previous total conductance value assuming the total GJ conductance is constant for $\Delta t=0.01 \mathrm{~ms}$. The expected value of the total conductance is

$$
\begin{aligned}
\mathrm{E}\left[G_{g j}\left(V_{j}\right)\right] & =\mathrm{E}\left[N G_{c h}\right] \\
& =N\left(p_{H H} G_{H H}+p_{H L} G_{H L}+p_{L H} G_{L H}\right)
\end{aligned}
$$

where the expectation of $G_{c h}$ is found by using the probability mass function of the single GJ channel conductance in (10). After simulating the AP propagation by using the expectation of $G_{g j}$ given in (21), we use the junctional voltage between $\mathrm{TC}$ and RC membranes for the channel capacity analysis.

An example of a successful and an failed transmissions of APs between TC and RC can be seen in Fig. 6 which is the graph of the membrane voltage changes of TC and $\mathrm{RC}$ over time. The channel propagation delay, denoted by $\tau$, is the delay between upstrokes of the action potentials generated in TC and RC. The AP pulse duration, denoted by $T_{A P}$, is the time duration between the AP initiation instant and the time that the membrane potential is restored to its initial value. The cycle length, denoted by $T_{C}$, is the time between two successive stimulus that are applied to TC. That is, $T_{C}$ is the signaling interval of TC. Therefore, the information transmission rate,

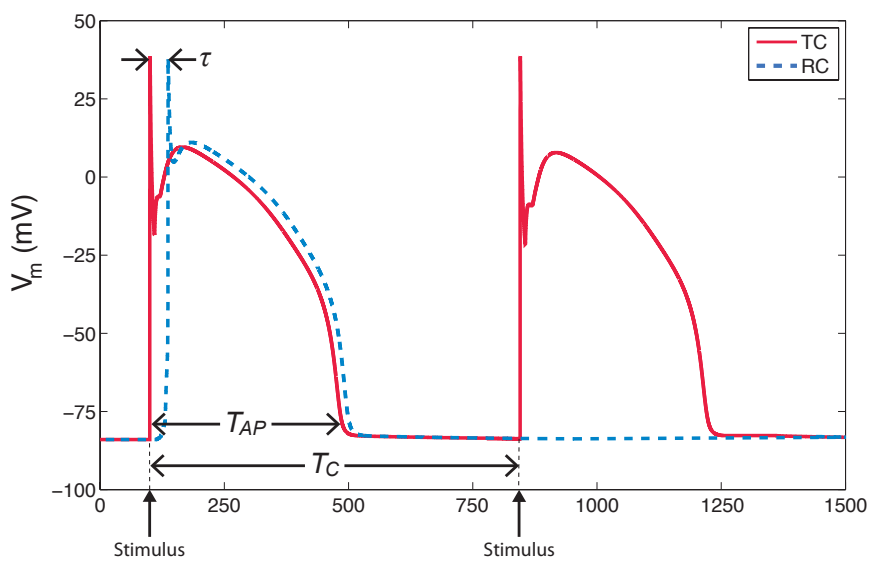

Time (msec)

Fig. 6. An illustration for the successful and the failed AP transmission between TC and RC. Time instants of two stimuluses that are applied to TC and the membrane potential changes of TC and RC are shown. The simulation parameters are $N=110, L=100 \mu \mathrm{m}, r=11 \mu \mathrm{m}, R_{i}=250 \Omega \mathrm{cm}, R_{e}=$ $100 \Omega \mathrm{cm}, R_{m}=20 \mathrm{k} \Omega \mathrm{cm}^{2}, \rho=100 \mathrm{n} M, P_{\max }=0.3, M=30000$, $n=1.6, T_{s}=T_{A P}, V_{e}=V_{i} / 5$ [26], [37], [40].

TABLE I

SimUlation PARAMETERS

\begin{tabular}{|l|l|l|}
\hline Parameter & Symbol & Value Range \\
\hline Number of GJ channels & $N$ & $105-120$ \\
\hline Cardiomyocyte length & $L$ & $90-105 \mu \mathrm{m}$ \\
\hline Cardiomyocyte radius & $r$ & $10.0-11.5 \mu \mathrm{m}$ \\
\hline Membrane resistivity & $R_{m}$ & $19-20 \mathrm{k} \Omega \mathrm{cm}^{2}$ \\
\hline Free $\mathrm{Ca}^{2+}$ concentration & $\rho$ & $590-600 \mathrm{nM}$ \\
\hline
\end{tabular}

denoted by $R$, is given by $R=1 / T_{C}$. In Fig. 6 , the channel propagation delay $\tau$, the AP pulse duration $T_{A P}$, and the cycle length $T_{C}$ can be seen.

The junctional voltage attained from the simulation is used to evaluate $p_{H H}, p_{H L}$, and $p_{L H}$ by using (7) and (8). Since the junctional voltage $V_{j}$ is time varying and the probabilities depend on $V_{j}$, time average of the probabilities are used for (12). Furthermore, the numerical analysis of the mutual information expression in (20) is performed with the parameters given in Table I. However, derivations of mathematical expressions for both the channel propagation delay $\tau$ and the action potential pulse duration $T_{A P}$ are too complex because of nonlinear and stochastic nature of the membrane ion channels generating the AP [26]. Therefore, the numerical values of $\tau$ and $T_{A P}$ are obtained from the simulation results of the AP generation model as performed in the physiological investigations [39]. The numerical analysis is conducted on MATLAB. For the numerical analysis, we keep the following parameters constant at their typical physiological values which are $R_{i}=250 \Omega \mathrm{cm}$, $R_{e}=100 \Omega \mathrm{cm}$ [40], $P_{\max }=0.3, M=30000, n=1.6$ [37]. Moreover, we assume $V_{i}=5 V_{e}$ and $T_{s}=T_{A P}$.

\section{A. Channel Capacity}

1) Effect of Number of GJ Channels: For the first analysis, we investigate the effect of the number of the GJ channels in the GJ, $N$, on the capacity of the GJ communication channel $C_{g j}$. The analysis is carried out for the GJ between TC and 

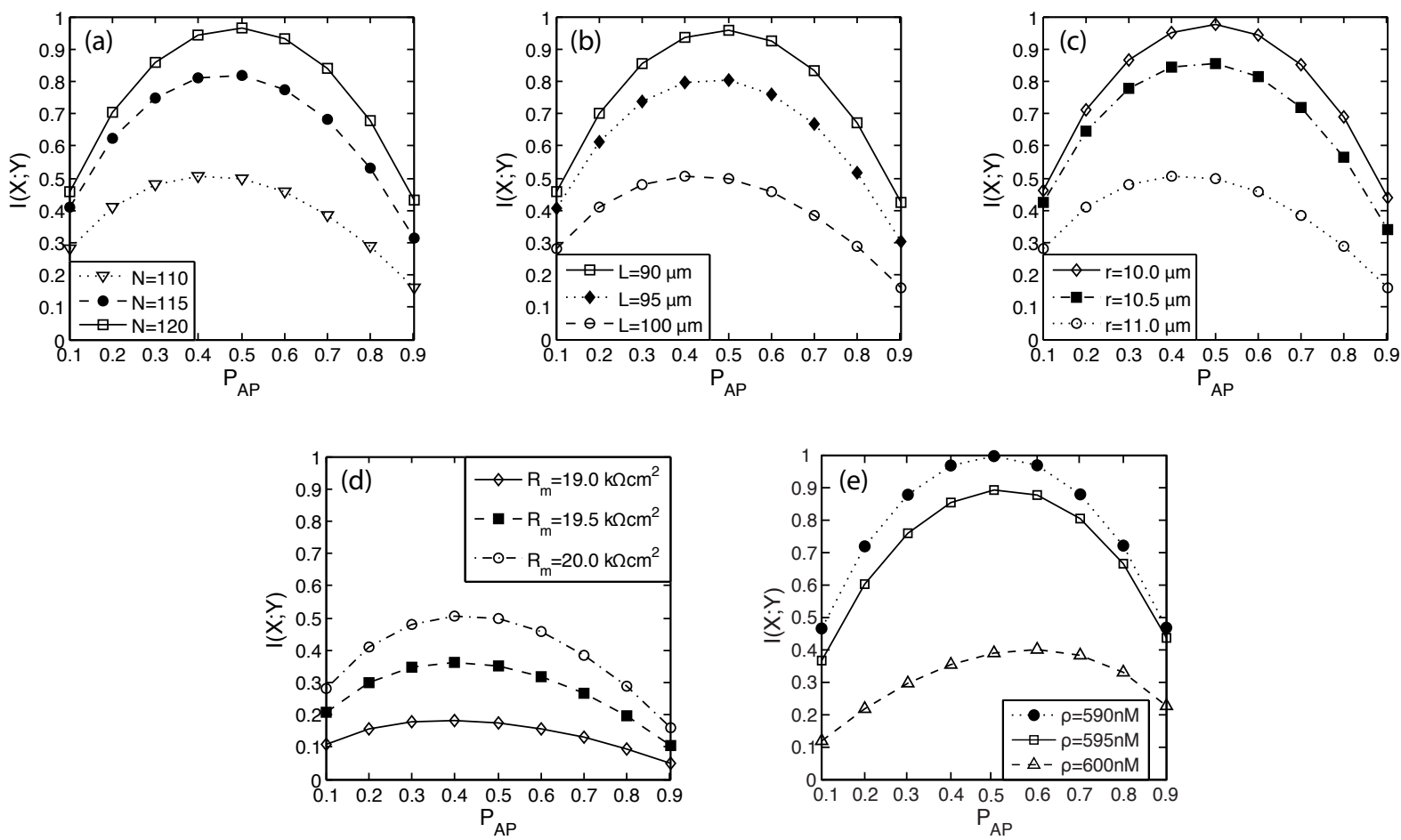

Fig. 7. $I(X ; Y)$ in bit for several (a) number of the GJ channels, $N$, (b) length of the cardiomyocyte, $L$, (c) radius of the cardiomyocyte, $r$, and (d) the membrane resistivity of the cardiomyocyte, $R_{m}$, (e) the free $\mathrm{Ca}^{2+}$ concentration in the cytoplasm, $\rho$, with varying $P_{A P}$.

$\mathrm{RC}$ for which number of the GJ channels are varied from $N=105$ to $N=120$. We set the rest of the parameters as $L=100 \mu \mathrm{m}, r=11 \mu \mathrm{m}, R_{m}=20 \mathrm{k} \Omega \mathrm{cm}^{2}$ and $\rho=100 \mathrm{nM}$ [26], [37], [40]. The critical GJ conductance value for the given parameter set is $G_{g j}^{*}=5.80 \mathrm{nS}$ found by using (3). In Fig. 7(a), the mutual information $I(X ; Y)$ given in $(20)$ is shown with varying AP generation probability $P_{A P}$ for different number of the GJ channels. Changing the number of the GJ channels in the GJ effects directly the conductance value of the junction. Therefore, less number of the GJ channels results in smaller conductance value which causes $P_{f}$ to become larger, which also can be seen in Fig. 3. In other words, less number of the GJ channels makes the transmission of bit 1 more erroneous; thus, the capacity of the GJ communication channel decreases accordingly as illustrated in Fig. 7(a). For $N=105$, the capacity is $C_{g j}=0.19 b i t /$ use attained with $P_{A P}=0.39$ and for $N=120$, the capacity is $C_{g j}=0.97 b i t / u s e$ attained with $P_{A P}=0.49$. Furthermore, for $\mathrm{N}$ greater than 120, the capacity saturates and becomes $C_{g j}=1 b i t / u s e$. Thus, the capacity of the GJ communication designed for nanodevices can be increased with an increase in the number of the GJ channels.

2) Effect of Cardiomyocyte Length: In this analysis, we examine the GJ communication channel capacity variation with respect to the length of cardiomyocytes, $L$. We change the value of $L$ while keeping the other parameters constant for $N=110, r=11 \mu \mathrm{m}, R_{m}=20 \mathrm{k} \Omega \mathrm{cm}^{2}$, and $\rho=$ $100 n M$ [26], [37], [40]. The change of the mutual information $I(X ; Y)$ with varying AP generation probability of TC for different $L$ values is shown in Fig. 7(b). The typical length of a ventricular cardiomyocyte is $L=100 \mu \mathrm{m}$ which leads to $C_{g j}=0.51 \mathrm{bit} /$ use reached with $P_{A P}=0.42$. For lengths larger than $100 \mu \mathrm{m}$, the critical conductance value increases as a result of (3). Thus, the increase in the critical conductance value yields increase in $P_{f}$ and hence dramatical decrease in the capacity. The capacity reduction due to increasing the length can be seen in Fig. 7(b). For $L=105 \mu m$, we find $C_{g j}=0.21 b i t / u s e$ reached with $P_{A P}=0.39$, and for $L=90 \mu \mathrm{m}, C_{g j}=0.96 \mathrm{bit} /$ use reached with $P_{A P}=0.49$. The result of this analysis shows that the GJ communication channel between shorter cardiomyocytes is higher than the capacity between longer cardiomyocytes. As a result, the capacity of the GJ communication used for the communication of nanodevices can be improved by using short nanodevices.

3) Effect of Cardiomyocyte Radius: Here, we investigate the effect of cardiomyocyte radius on the GJ communication channel capacity by changing the value of $r$, while setting the other parameters as $N=110, L=100 \mu \mathrm{m}, R_{m}=20 \mathrm{k} \Omega \mathrm{cm}^{2}$ and $\rho=100 n M$ [26], [37], [40]. In order to understand change of the channel capacity with respect to $r$, we compute $I(X ; Y)$ given in (20) for different radius values with varying AP generation probability. The result of the analysis is shown in Fig. 7(c). The capacity decreases significantly with increasing cell radius. However, decreasing the radius, for example, to $r=10 \mu \mathrm{m}$ gives $C_{g j}=0.98 \mathrm{bit} /$ use attained with $P_{A P}=0.49$. Also, increasing to $r=11.5 \mu \mathrm{m}$ gives $C_{g j}=0.16 b i t / u s e$ attained with $P_{A P}=0.38$. As a result, the GJ communication channel between thiner cardiomyocytes has larger capacity compared to thicker cardiomyocytes. The result of this analysis shows that using thin nanodevices can increase 


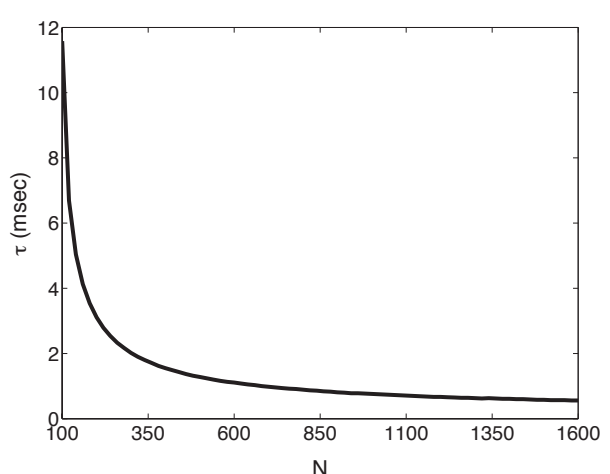

Fig. 8. The channel propagation delay, $\tau$, between $\mathrm{TC}$ and $\mathrm{RC}$ with respect to the number of the GJ channels in the GJ, $N$.

the capacity the GJ communication between nanodevices.

4) Effect of Membrane Resistivity Cardiomyocyte: In this analysis, we investigate the effect of the membrane resistivity, $R_{m}$, of cardiomyocytes on the GJ communication channel capacity. The membrane resistivity plays a critical role for the AP initiation and the propagation as described in the Section II. For the numerical analysis, only $R_{m}$ value is changed and the other parameters are set as $N=110, L=100 \mu \mathrm{m}$, $r=11 \mu m$ and $\rho=100 n M$ [26], [37], [40]. Increasing $R_{m}$ decreases the critical conductance value given in (3); therefore, increasing $R_{m}$ yields reduction in $P_{f}$ expressed in (13) and hence increase in the channel capacity. The increase in the channel capacity because of increase in $R_{m}$ can be seen in Fig. 7(d). The results of the numerical analysis show that for $R_{m}=19 \mathrm{k} \Omega \mathrm{cm}^{2}, C_{g j}=0.18 \mathrm{bit} /$ use achieved with $P_{A P}=0.38$ and for $R_{m}=20 \mathrm{k} \Omega \mathrm{cm}^{2}, C_{g j}=0.51 \mathrm{bit} / \mathrm{use}$ achieved with $P_{A P}=0.42$. As a result, the GJ communication between cardiomyocytes having higher membrane resistivity also has higher channel capacity. Furthermore, the capacity of the GJ communication designed for nanodevices can be increased by using membranes with high resistivity values.

5) Effect of Free Calcium Concentration: In this part, we investigate the effect of the free $\mathrm{Ca}^{2+}$ concentration $\rho$ in the cytoplasm of cardiomyocytes on the GJ communication channel capacity. In this analysis, we use the parameter values as $N=1000, L=100 \mu \mathrm{m}, r=11 \mu \mathrm{m}$ and $R_{m}=20 \mathrm{k} \Omega \mathrm{cm}^{2}$ [26], [40]. In Fig. 7(e), the mutual information $I(X ; Y)$ is shown for different concentration $\rho$ values. An increase in the free $\mathrm{Ca}^{2+}$ concentration increases the probability of the spontaneous AP wave initiation $P_{w}$ as stated in Section II-D. Therefore, increasing the free $\mathrm{Ca}^{2+}$ concentration decreases the capacity of the GJ communication channel $C_{g j}$ given in (21). For example, we find $P_{w}=2.98 \times 10^{-4}$ and $C_{g j}=0.99 \mathrm{bit} /$ use for $\rho=590 \mathrm{nM}$ and $P_{w}=0.41$ and $C_{g j}=0.40 \mathrm{bit} /$ use for $\rho=600 \mathrm{nM}$. That is, if fluctuations in the free $\mathrm{Ca}^{2+}$ concentration result in $\rho>590 \mathrm{nM}$, the capacity of the GJ communication channel is reduced significantly. Thus, we can say that high $\mathrm{Ca}^{2+}$ concentration in the cytoplasm causes more erroneous transmission of the information due to increased $P_{w}$.

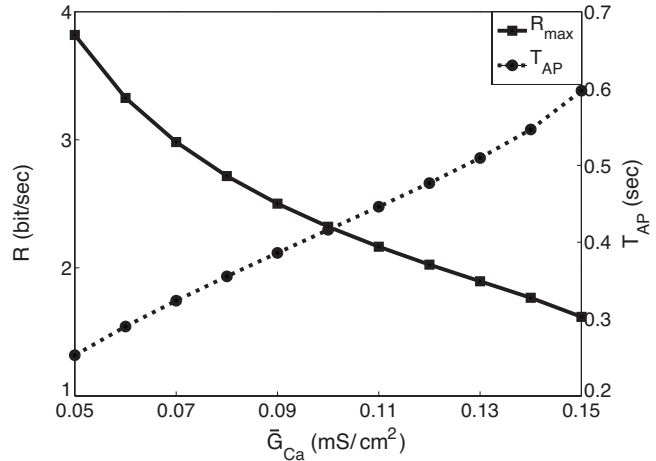

Fig. 9. The information transmission rate, $R$, and the AP duration, $T_{A P}$, with respect to the maximum conductance of the calcium channels, $G_{C a}$.

\section{B. Channel Propagation Delay}

An AP pulse transmitted by $\mathrm{TC}$ to $\mathrm{RC}$ propagates through the GJ channels in the GJ between TC and RC. During the AP propagation, the membrane voltage change with respect to time is shown in Fig. 6. As illustrated in the figure, there is a propagation delay $\tau$ of the GJ communication channel. In Fig. 8, variation of the propagation delay with respect to the number of the GJ channels is shown. We use the typical physiological values for the remaining parameters which are $L=100 \mu m, r=11 \mu m, R_{m}=20 k \Omega \mathrm{cm}^{2}$ and $\rho=100 \mathrm{nM}$ [26], [37], [40]. The delay of AP propagation from TC to RC decreases with increasing the number of the GJ channels. We find $\tau=11.58 \mathrm{~ms}$ for $N=100$, and $\tau=0.56 \mathrm{~ms}$ for $N=1600$. Furthermore, the delay shows an abrupt decrease for increasing $N$ from 100 to 300, and further increasing $N$ causes the delay decrease slowly. For $N$ less than 300, the coupling between TC and RC is so weak that increasing $N$ leads large decrease in $\tau$. On the other hand, for $N$ larger than 300, TC and RC are well coupled so that increasing $N$ slightly decreases $\tau$. Consequently, we can say that further increasing $N$ saturates the channel propagation delay to the propagation delay of the cytoplasm of cardiomyocyte, i.e., the effect of discreteness of the cardiac fiber due to the GJ connections diminishes.

\section{Information Transmission Rate}

We model the GJ communication between two cardiomyocytes, i.e., TC and RC, as a binary digital communication with OOK modulation as stated previously. Assuming the cycle length is the same as the AP duration, i.e., $T_{C}=T_{A P}$, the information transmission rate, $R$, in $b i t / s e c$ for the GJ communication is given as $R=\frac{1}{T_{A P}}$ where $T_{C}$ and $T_{A P}$ are the cycle length and AP duration, respectively, as shown in Fig. 6. The AP generation in a cardiomyocyte is achieved by the voltage dependent conductance of the ion channels embedded in the membrane as explained in Section II-A. In Fig. 9, $T_{A P}$ and $R$ with respect to the maximum conductance of the calcium channels, denoted by $\bar{G}_{C a}$, is shown. $T_{A P}$ variation for different $\bar{G}_{C a}$ values is found from the simulation results of the cardiac AP model in [26] and the variation of $R$ for different $\bar{G}_{C a}$ values is obtained accordingly. In our 
previous analysis, we use the typical physiological value of $G_{C a}=0.09 \mathrm{mS} / \mathrm{cm}^{2}$ [26]. Furthermore, in this part, we fix the other model parameters as $N=1000, L=100 \mu \mathrm{m}$, $r=11 \mu \mathrm{m}, R_{m}=20 \mathrm{k} \Omega \mathrm{cm}^{2}$, and $\rho=100 \mathrm{nM}$ [26], [37], [40]. According to Fig. 9, $T_{A P}$ increases and $R$ decreases with increasing $\bar{G}_{C a}$. We find $T_{A P}=259 \mathrm{msec}$ and $R=$ $3.86 \mathrm{bit} / \mathrm{sec}$ for $\bar{G}_{C a}=0.05 \mathrm{mS} / \mathrm{cm}^{2}$ and $T_{A P}=598 \mathrm{msec}$ and $R=1.67 \mathrm{bit} / \mathrm{sec}$ for $\bar{G}_{\mathrm{Ca}}=0.15 \mathrm{mS} / \mathrm{cm}^{2}$. The analysis presented in [41] also shows that reduced permeability of the calcium channels results in shorter AP durations which confirms our results. Moreover, since we analyze $R$ for $N=130$ which results in $C_{g j}=1 b i t$, the transmission rate of TC is the same as the reception rate of RC. Although increasing $R$ is a desired objective in the classical communication, for the GJ communication of cardiomyocytes, increased $R$ due to reduced $\bar{G}_{C a}$ may cause several arrhythmias which are discussed in the next section.

\section{Gap Junction COMmunication Channel PARAMETERS AND CARDiAC DiseASES}

The GJ communication between cardiomyocytes enables the electrical signal conduction, i.e., the AP propagation, in the heart. Synchronous propagation of the APs leads the heart to contract and relax as a single unit. Therefore, the GJ communication channel failures and deficiencies cause several disorders in synchronous propagation of the APs and hence arrhythmias in the heart. In this section we investigate the relations between cardiac diseases and the GJ communication parameters which are the channel capacity, the channel propagation delay and the information transmission rate.

\section{A. Channel Capacity and Cardiac Diseases}

In this part, we investigate the relation between cardiac diseases and the channel capacity of the GJ communication. In the previous section, the effects of several parameters on the channel capacity are shown. For this part, we present the relation between these parameters and cardiac diseases using the results of several medical researches. For each parameter, the relation between the channel capacity and cardiac diseases is observed as follows.

1) Number of Gap Junction Channels: The numerical analysis performed in the previous section shows that decreased GJ coupling due to reduction in the total number of the GJ channels in the GJ causes capacity of the channel to fall. According to results of investigations of heart diseases, reduced GJ coupling leads to several heart diseases such as sudden death due to spontaneous ventricular arrhythmia [42] or increased incidence of ventricular tachycardias [43]. We can conclude that the reduction in the capacity of the channel due to reduced GJ coupling is related with increased incidence of cardiac diseases mentioned above. That is, reasons of these heart diseases cause also the channel capacity to decrease. Therefore, the channel capacity can be used in investigation and diagnosis of cardiac diseases discussed end of this section.

2) Length of Cardiomyocyte: The GJ communication the channel capacity decreases with increasing the length of the cardiomyocyte, $L$, as shown in Fig. 7(b). The results presented in [44] which investigates effects of structural properties of cardiomyocyte on ischemic cardiomyopathy (ICM) show that length of cardiomyocytes is significantly longer in patients with ICM. We can say that reduction in the capacity of the GJ channel causes more erroneous transmission of the information; as a result, coordination between cardiomyocytes decreases and the cardiac tissue becomes diseased. It can be concluded that decrease in the GJ communication channel capacity due to increased $L$ and increased occurrence of ICM due to longer cardiomyocytes is correlated.

3) Radius of Cardiomyocyte: From the numerical analysis, we know that the channel capacity of the GJ communication decreases with increasing the radius of cardiomyocytes as illustrated in Fig. 7(c). The results of the study presented in [45] reveal that the radius of cardiomyocytes is significantly higher in hypertensive patients compared to control subjects. In this case, increase in the radius of cardiomyocytes causes both decrease in the channel capacity and increased occurrence of hypertensive disease. Therefore, the channel capacity decrease due to increased cell radius is correlated with increased incidence of the cardiac diseases.

4) Membrane Resistivity: The numerical analysis shows that reduction in the membrane resistivity decreases the channel capacity of the GJ communication as seen in Fig. 7(d). According to an analysis presented in [46], decrease of the cardiomyocyte membrane resistivity is related to the impairment of impulse propagation which can cause severe cardiac arrhythmias. This relation also confirms our result since according to (3), decreasing $R_{m}$ increases the critical conductance value which can lead to the AP propagation impairment. Consequently, reduction in the channel capacity due to reduced membrane resistivity is accompanied with cardiac arrhythmia.

5) Free Calcium Concentration: From the numerical analysis, we observe that the GJ communication channel capacity decreases with an increase in the free $\mathrm{Ca}^{2+}$ concentration inside the cytoplasm of the cardiomyocytes as seen in Fig. 7(e). Free $\mathrm{Ca}^{2+}$ overload causes several triggered arrhythmias which are delayed-afterdepolarizations (DADs) and earlyafterdepolarization (EADs) [47]. Furthermore, these triggered arrhythmias are major initiators of ventricular tachycardia which is an immediate precursor of ventricular fibrillation and a major cause of sudden death in heart failure [47]. We can conclude that the GJ communication channel capacity reduction due to increased free $\mathrm{Ca}^{2+}$ concentration inside the cytoplasm is correlated with the increased occurrence of the ventricular tachycardia.

\section{B. Channel Propagation Delay and Cardiac Diseases}

According to the results of the analysis of the AP propagation delay through the GJ communication channel, increasing the number of the GJ channels decreases the propagation delay as shown in Fig. 8. However, decreasing $N$ causes the propagation delay to increase accompanied with decrease in the channel capacity. That is, decreasing the number of the GJ channels increases the propagation delay and decreases the channel capacity. As stated above, the reduction of the GJ 
coupling due to decreasing the number of GJ channel $N$ causes to several cardiac diseases. Therefore, it can be concluded that the AP propagation delay between cardiomyocytes is higher for cases of spontaneous ventricular arrhythmia [42] and ventricular tachycardia [43].

\section{Information Transmission Rate and Cardiac Diseases}

The results of the numerical analysis show that decreasing the maximum calcium channel conductance, $\bar{G}_{C a}$, increases the information transmission rate between TC and RC. Although in classical communication, increasing $R$ is a desired objective, physiological properties of cardiomyocytes cannot support very high transmission rates. In other words, at higher transmission rates, each received AP pulse also leads receiver cardiomyocyte to contract and relax at higher rates due to excitation-contraction coupling property of cardiomyocytes. Therefore, since heart cannot support very high beating rates, increasing $R$ causes several arrhythmias. In [48], heart rates $>100$ beats/min are referred as tachycardia causing heart to be arrhythmic. Furthermore, prolongation of the AP duration, $T_{A P}$, is a reason of the development of early afterdepolarizations which cause a number of arrhythmias in the heart including long-QT syndrome and heart failure [36], [49]. As a result, we can say that both very low $R$ and very high $R$ due to increased and decreased $T_{A P}$, respectively, increase occurrence of cardiac diseases stated above.

\section{Application on Nanomedicine}

Nanomedicine is the application of molecular nanotechnology on medicine and availability of such technologies brings dramatic progress in the medical services [50]. Investigation, prediction, diagnosis and treatment of diseases are some areas that are covered by nanomedicine. To be able to perform these applications, nanomedicine proposes the use of nanodevices, e.g., nanosensors, nanovehicles, and nanoactuators [50], [51].

The most important deductions inferred from the observations given in this section are those in diseased myocardium, the channel capacity of the GJ communication is lower and the propagation delay is higher than for the case of healthy myocardium. Furthermore, the causes of very high or very low information transmission rates contribute occurrence of cardiac diseases. As a result, the information theoretical analysis of the GJ communication may provide valuable insights into causes of cardiac diseases. Moreover, the channel capacity, the channel propagation delay and the transmission rate can be used as metrics for investigation, prediction, diagnosis and treatment of several cardiac diseases in nanomedicine [8]. These parameters can be measured and monitored by using multiple intra-body nanodevices and nanosensors communicating with cardiomyocytes via GJ channels. For instance, in [52], the use of nanoparticles for molecular imaging, advanced therapeutics and drug delivery systems in treatment of cardiovascular diseases is discussed. In addition, the information theoretical analysis of the GJ communication can be used for simulation tools of drug tests to confirm reliability of drugs. Hence, diagnosis and treatment techniques of cardiac diseases using the information theoretical model of the GJ communication stands as a promising application of emerging nanomedicine.

\section{CONCLUSION}

In this study, we model the GJ communication channel between cardiomyocytes from the information theoretic perspective for the first time in the literature. Firstly, we introduce the AP propagation between $\mathrm{TC}$ and $\mathrm{RC}$ and for the success or failure of the propagation. Afterwards, we describe the stochastic GJ channel model. Then, as a result of the information theoretic analysis, we derive a closed form expression for the capacity of the channel between TC and RC. Finally, the capacity of the channel is analyzed numerically by using the capacity expression for different physiological parameters, e.g., the number of the GJ channels, the cell length, the cell radius, and the membrane resistivity. The results of the numerical analysis of the channel capacity and the propagation delay show that decrease in the channel capacity and increase in the propagation delay are highly correlated to several cardiac diseases such as spontaneous ventricular arrhythmia or ischemic cardiomyopathy. It is also observed that very high or very low information transmission rates also causes cardiac arrhythmias. Consequently, the model of the communication channel between cardiomyocytes presented in this paper can be used to get a new insight into reasons of cardiac diseases and hence diagnose and treat these diseases with advances in nanomedicine. Furthermore, the numerical results reveal that the capacity of the GJ communication channel can be increased by selecting appropriate parameter values. Thus, the capacity of the GJ communication designed for the communication of nanodevices can be improved using the GJ communication model presented in this study. Moreover, our ongoing works aim to investigate the GJ communication for multiple cells in a 2D and 3D network of cardiomyocytes.

\section{REFERENCES}

[1] S. Hiyama, Y. Moritani, T. Suda, R. Egashira, A. Enomoto, M. Moore and T. Nakano, "Molecular Communication," in Proc. of NSTI Nanotech. 2005, Anaheim, California, USA, 2005.

[2] M. Gregori, I. F. Akyildiz, "A new nanonetwork architecture using flagellated bacteria and catalytic nanomotors," IEEE JSAC, vol. 28, no. 4, pp. 612-619, 2010.

[3] B. Atakan, S. Balasubramaniam, O. B. Akan, "Body Area NanoNetworks with Molecular Communications in Nanomedicine," IEEE Com. Mag., vol. 50, no. 1, pp. 28-34, January 2012

[4] I. F. Akyildiz, F. Brunetti, C. Blazquez, "Nanonetworks: A new communication paradigm," Computer Networks (Elsevier), vol. 52, no. 12, pp. 2260-2279, 2008.

[5] L. P. Giné and I. F. Akyildiz, "Molecular communication options for long range nanonetworks," Computer Networks (Elsevier), vol. 53, no. 16, pp. 2753-2766, Nov 2009.

[6] M. Pierobon, I. F. Akyildiz, "A Physical End-to-End Model for Molecular Communication in Nanonetworks," IEEE JSAC, vol. 28, no. 4, pp. 602 611, May 2010.

[7] M. Pierobon, I. F. Akyildiz, "Diffusion-based Noise Analysis for Molecular Communication in Nanonetworks," IEEE Transactions on Signal Processing, vol. 59, no. 6, pp. 2532-547, June 2011.

[8] D. Malak, O. B. Akan, "Molecular communication nanonetworks inside human body," Nano Communication Networks, vol. 3, pp. 19-35, 2012.

[9] B. Atakan, O. B. Akan, "Deterministic Capacity of Information Flow in Molecular Nanonetworks," Nano Communication Networks, vol. 1, no. 1, pp. 31-42, March 2010.

[10] M. Pierobon, I. F. Akyildiz, "Information Capacity of Diffusion-based Molecular Communication in Nanonetworks," in Proc. of IEEE Int. Conf. on Comp. Comm, INFOCOM, Miniconf., China, April 2011. 
[11] A. Eckford, "Nanoscale communication with brownian motion," in Proc. 41st Annu. Conf. Inf. Sci. Syst., 2007, pp. 160-165.

[12] P. J. Thomas et al., "The diffusion mediated biochemical signal relay channel," in Proc. 17th Annu. Conf. Neural Inf. Process. Syst., 2003.

[13] B. Atakan, O. B. Akan, "An information theoretical approach for molecular communication," in Proc. 2nd Int. Conf. Bio-Inspired Models Netw., Inf., Comput. Syst., 2007, pp. 33-40.

[14] B. Atakan, O. B. Akan, "On molecular multiple-access, broadcast, and relay channels in nanonetworks," in Proc. 3rd Int. Conf. Bio-Inspired Models Netw., Inf., Comput. Syst., 2008, Art. no. 16.

[15] T. Nakano, J. Q. Liu, "Design and analysis of molecular relay channels: An information theoretic approach," IEEE Transactions on Nanobioscience, vol. 9, no. 3, pp. 213-221, Jun 2009.

[16] N. M. Kumar, N. B. Gilula, "The gap junction communication channel," Cell, vol. 84, pp. 381-388, 1996

[17] T. Nakano et al., "Molecular Communication through Gap Junction Channels," Springer Transactions on Computational Systems Biology X, vol. 5410, pp. 81-99, 2008.

[18] T. Nakano et al., "Molecular communication for nanomachines using intercellular calcium signaling," in Proc. of the 5th IEEE Conf. on Nanotechnology, vol. 2, pp. 478-481, Nagoya, Japan, 2005

[19] M. Kuscu, O. B. Akan, "A Physical Channel Model and Analysis for Nanoscale Molecular Communications with Förster Resonance Energy Transfer (FRET)," IEEE Transactions on Nanotechnology, vol. 11, no:1, pp 200-207, 2012.

[20] B. J. MacLennan, "Morphogenesis as a model for nano communication," Nano Communication Networks, vol. 1, no. 3, pp. 199-208, 2010.

[21] H. Niessen et al., "Selective permeability of different connexin channels to the second messenger inositol 1,4,5-triphosphate," J. Cell Sci., vol. 113, pp. $1365-1372,2000$

[22] G. Sohl, K. Willecke, "Gap junctions and the connexin protein family," Cardiovasc. Res., vol. 62, pp. 228-232, 2004.

[23] D. A. Jaye, Y. Xiao, D. C. Sigg, "Basic Cardiac Electrophysiology: Excitable Membranes," in Cardiac Electrophysiology Methods and Models, D. C. Sigg, P. A. Iaizzo, Y. Xiao, B. He, Ed. Springer, 2010, pp. 41-51.

[24] A. P. de Carvalho, B. F. Hoffman, M. de P. Carvalho, "Two components of the cardiac action potential. I. Voltage-time course and the effect of acetylcholine on atrial and nodal cells of the rabbit heart," J. Gen. Physiol., vol. 54, pp. 607-635, 1969.

[25] A. L. Hodgkin, A. F. Huxley, "A quantitative description of membrane current and its application to conduction and excitation in nerve," $J$ Physiol., vol. 117, pp. 500-544, 1952.

[26] C. H. Luo, Y. A. Rudy, "A dynamic model of the cardiac ventricular action potential. I. Simulations of ionic currents and concentration changes," Circ. Res., vol. 74, pp. 1071-1096, 1994.

[27] C. H. Luo, Y. A. Rudy, "A dynamic model of the cardiac ventricular action potential. II. Afterdepolarizations, triggered activity, and potentiation," Circ. Res., vol. 74, pp. 1097-1113, 1994.

[28] J. P. Keener, "The effects of discrete gap junction coupling on propagation in myocardium," J. Theor. Biol., vol. 148, pp. 49-82, 1991.

[29] G. W. Beeler, H. Reuter, "Reconstruction of the action potential of myocardial fibres,” J. Physiol. (Lond.), vol. 268, pp.177-210, 1977.

[30] L. Ebihara, E. A. Johnson, "Fast sodium current in cardiac muscle, a quantitative description,” Biophys. J., vol. 32, pp. 779-790, 1980.

[31] S. Dhein, Cardiac Gap Junctions: Physiology, Regulation, Pathophysiology and Pharmacology, Karger, Basel, 1998.

[32] S. Baigent, S. Jaroslav, A. Warner, "Modeling the effect of gap junction nonlinearities i systems of coupled cells," J. Theor. Biol., vol. 186, pp. 223-239, 1997.

[33] F. F. Bukauskas et al., "Gating properties of gap junction channels assembled from connexin43 and connexin43 fused with green fluorescent protein," Biophys. J., vol. 81, pp. 137-152, 2001.

[34] V. Valiunas et al., "Formation of heterotypic gap junction channels by connexins 40 and 43," Circ. Res., vol. 86, pp. E42-E49, 2000.

[35] H. Z. Wang, J. Li, L. F. Lemanski, and R. D. Veenstra, "Gating of mammalian cardiac gap junction channels by transjunctional voltage," Biophys. J., vol. 63, pp. 139-151, 1992

[36] H. B. Nuss et al., "Cellular basis of ventricular arrhythmias and abnormal automaticity in heart failure," Am. J. Physiol., vol. 277, pp. H80-H91, 1999.

[37] L. T. Izu et al., "Evolution of Cardiac Calcium Waves from Stochastic Calcium Sparks," Biophysical J., vol. 80, pp. 103-120, Jan. 2001.

[38] C. E. Shannon, "A mathematical theory of communication," The Bell System Technical Journal, vol. 27, pp. 379-423, 623-656, Jul 1948.

[39] S. Rohr, "Role of gap junctions in the propagation of the cardiac action potential," Cardiovasc. Res., vol. 62, pp. 309-322, 2004.
[40] M. S. Spach, J. F. Heidlage, P. C. Dolber, and R. C. Barr, "Extracellular discontinuities in cardiac muscle: evidence for capillary effects on the action potential foot," Circ. Res., vol. 83, pp. 1144-1164, 1998.

[41] J. J. Fox, J. L. McHarg, and R. F. Gilmour, "Ionic mechanism of cardiac alternans," Am. J. Physiol., vol. 282, pp. H516-H530, 2002.

[42] D. E. Gutstein et al., "Conduction slowing and sudden arrhythmic death in mice with cardiac-restricted inactivation of connexin43," Circ. Res. vol. 88, pp. 333-339, 2001.

[43] D. L. Lerner, K. A. Yamada, R. B. Schuessler, J. E. Saffitz, "Accelerated onset and increased incidence of ventricular arrhythmias induced by ischemia in Cx43-deficient mice," Circ., vol. 101, pp. 547-552, 2000.

[44] A. M. Gerdes, et al., "Structural remodeling of cardiac myocytes from patients with chronic ischemic heart disease," Circ., vol. 86, pp. 426-430, 1992.

[45] K. Amann et al., "Myocyte/capillary mismatch in the heart of uremic patients," J. Am. Soc. Nephrol., vol. 9, pp. 1018-1022, 1998.

[46] W. C. De Mello, "Cell swelling, impulse conduction, and cardiac arrhythmias in the failing heart. Opposite effects of angiotensin II and angiotensin (1-7) on cell volume regulation," Mol. Cell. Biochem., vol. 330, pp. 211-217, 2009.

[47] D. M. Bers, "Calcium and cardiac rhythms: Physiological and pathophysiological," Circ.Res., vol. 90, pp. 14-17, 2002.

[48] J. T. Nguyen, X. Li, and F. Lu, "The Electrocardiogram and Clinical Cardiac Electrophysiology," in Cardiac Electrophysiology Methods and Models, D.C. Sigg, et al., Ed. Springer, 2010, pp. 91-116.

[49] J. K. Donahue, M. Strom, and I. D. Greener, "Introduction to Translational Research," in Cardiac Electrophysiology Methods and Models, D.C. Sigg, et al., Ed. Springer, 2010, pp. 441-455.

[50] R. A. Freitas Jr., "Nanomedicine, vol. I: Basic Capabilities," Landes Bioscience, 1999.

[51] S. M. Moghimi et al., "Nanomedicine: current status and future prospects," The FASEB Journal, vol. 19, pp. 311-330, 2005.

[52] B. Godin et al., "Emerging applications of nanomedicine for the diagnosis and treatment of cardiovascular diseases," Trends Pharmacol. Sci. vol. 31, 199-205, 2010.

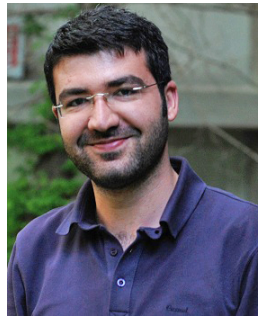

Deniz Kilinc received his B.Sc. degree in Electrical and Electronics Engineering from Middle East Technical University, Ankara, Turkey, in 2011. He is currently a research assistant in the Next-generation and Wireless Communication Laboratory and pursuing his M.Sc. degree at the Electrical and Electronics Engineering Department, Koc University, Istanbul, Turkey. His current research interests include nanoscale communication, intra-body molecular communication, and detection and estimation theory.

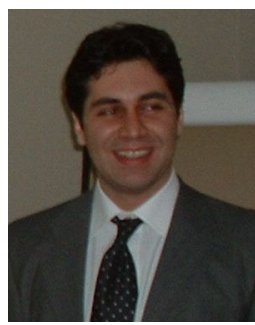

Ozgur B. Akan [M'00, SM'07] (akan@ku.edu.tr) received his Ph.D. degree in electrical and computer engineering from the Broadband and Wireless Networking Laboratory, School of Electrical and Computer Engineering, Georgia Institute of Technology in 2004. He is currently a full professor with the Department of Electrical and Electronics Engineering, Koc University and the director of the Next-generation and Wireless Communications Laboratory. His current research interests are in wireless communications, nano-scale and molecular communications, and information theory. $\mathrm{He}$ is an Associate Editor of IEEE Transactions on Vehicular Technology, International Journal of Communication Systems (Wiley), and Nano Communication Networks Journal (Elsevier). He is currently General Co-Chair of ACM MOBICOM 2012, IEEE MoNaCom 2012, and TPC Co-Chair of IEEE ISCC 2012. 